\title{
PERCEPCIÓN DE LOS ESTUDIANTES UNIVERSI- TARIOS SOBRE LA UTILIDAD DEL AULA VIRTUAL PARA EL APRENDIZAJE
}

Mirtha Ramos Arones

\section{Resumen}

El objeto de esta investigación fue identificar la percepción que tienen los estudiantes universitarios sobre la utilidad de las aulas virtuales como apoyo para sus aprendizajes. La metodología utilizada contempla un estudio descriptivo transversal, realizado mediante la aplicación de un cuestionario administrado a los estudiantes de las facultades de Ciencias Económicas y Empresariales e Ingeniería. Entre los resultados se evidencia que el 45\% de la población encuestada considera el A V como poco útil en sus procesos de aprendizaje; se ha encontrado relación entre la percepción que tienen los estudiantes sobre el A V. y el tipo de facultad a la que pertenecen, pero no se encontró relación entre el uso que hacen los docentes del A V. y el tipo de facultad a la que pertenecen los estudiantes.

Palabras clave: aula virtual, percepción, enseñanza-aprendizaje

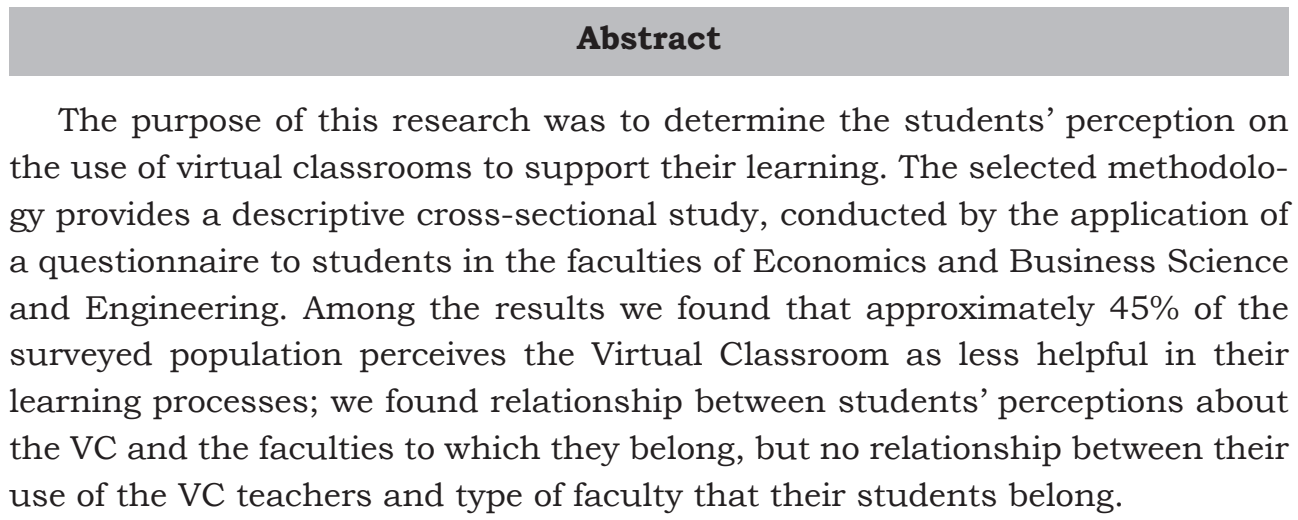


Keywords: virtual classroom, perception, usefulness, teaching-learning.

\section{INTRODUCCIÓN}

El desarrollo tecnológico actual nos está situando en un nuevo paradigma de enseñanza: las universidades de hoy se reconocen por la calidad de sus docentes para enfrentarse a los cambios permanentes que demanda el mundo globalizado. Estos aspectos exigen la presencia de docentes con formación interdisciplinar, que den lugar a nuevas y renovadas visiones metodológicas, nuevos roles docentes, más centrada ahora en el diseño curricular, en la gestión administrativa y en el uso racional y adecuado de los medios disponibles, especialmente de las tecnologías de información y comunicación (TIC).

Las TIC se pueden aprovechar para motivar el aprendizaje de los estudiantes. Para ello, una opción son las aulas virtuales, las cuales permiten crear espacios de aprendizaje asíncronos en los que se pueden realizar actividades de colaboración, de reflexión y de critica, con el fin de promover experiencias de aprendizaje relevantes. En la primera parte de esta investigación se desarrolla el marco teórico que permite contextualizar el proceso de aprendizaje apoyado con herramientas tecnológicas, específicamente A V. Se describen las características principales de esta herramienta, las ventajas, las responsabilidades que tienen las instituciones al implementarlas y la participación de los estudiantes en su uso.
Este estudio se enfocó primero en determinar la percepción que tienen los estudiantes sobre la utilidad del Aula Virtual para el aprendizaje; segundo, en conocer si los estudiantes encuentran en esta tecnología una ayuda académica, debido a que está diseñada en gran medida para ellos $\mathrm{y}$, tercero, en opinión de los estudiantes, cuál es el uso que hacen los docentes del AV Los resultados en tablas permiten evidenciar los principales aspectos con la temática evaluada. Finalmente, se estructuraron algunas conclusiones.

\section{MARCO TEÓRICO}

\section{E1 Aula Virtual}

El Aula Virtual está basado en una plataforma web llamada Moodle (Modular Object Oriented Distance) que fue creada por Martin Dougiamas, quien fue administrador de WebCT en la Universidad Tecnológica de Curtin. Moodle es una aplicación a la que se accede por medio de un navegador (Mozilla Firefox, Microsoft Internet Explorer, Opera, etc.), esto quiere decir que, para utilizar Moodle, es necesario una computadora con, al menos, un navegador web instalado y con conexión a Internet.

Moodle es una plataforma de software libre; la usan diferentes universidades e instituciones de educación superior, adaptando sus capacidades a las necesidades y preferencias de cada una de ellas, y creando así un entorno virtual de aprendizaje propio, 
que facilita la creación de entornos de enseñanza-aprendizaje, integrando materiales didácticos y herramientas de comunicación, colaboración y gestión educativas (Escalona, P. Expósito, E. y García, R. 2013).

\section{Moodle como soporte a las aulas vir- tuales de aprendizaje}

Moodle basa su diseño en las ideas de la pedagogía constructivista con énfasis en las teorias social cognitivas; parte de la premisa de que el conocimiento se construye en la mente del estudiante en lugar de ser solo transmitido, siendo la participación activa del estudiante una condición necesaria para el aprendizaje. El estudiante debe planificar, revisar y reflexionar $\mathrm{y}$, finalmente, desarrollar, innovar $\mathrm{y}$ crear el conocimiento, mientras que la función del profesor es la de facilitador, posibilitando el aprendizaje colaborativo. Son los estudiantes los que deben, a partir de la presentación de problemas del profesor, producir y generar su conocimiento (Sánchez, J. y Ramos, F. 2012).

\section{Moodle comúnmente denominada Aula Virtual.}

El Aula Virtual es un entorno telemático en página web, plataforma o software de enseñanza y aprendizaje mediado por la computadora. Está integrada y organizada de muchos recursos digitales de textos, imagen, sonido y animación, que permiten el desarrollo de las actividades de enseñanza. A diferencia de otra herramienta informática de apoyo a la enseñan$z a$, permite al estudiante, de manera instantánea, acceder desde cualquier computadora conectada a la red e ingresar al sitio donde se encuentran los materiales de estudio y realizar las actividades diseñadas por el profesor, es decir: realizar ejercicios, formular preguntas al docente sobre aspectos que no han quedado claros en clase; trabajar en equipo, utilizar herramientas de interacción como foros de discusión, charlas en directo y correo electrónico, todo ello de forma simulada, sin que medie una interacción física entre docentes y discentes. (Morales, L. y Guevara, J. 2009).

\section{Aulas Virtuales como apoyo a la educación presencial}

El verdadero reto de la educación no está en la innovación tecnológica sino en la innovación pedagógica, que deberá incluir el uso de las herramientas didácticas más apropiadas, entre ellas el AV, para diseñar actividades de aprendizaje de calidad para los estudiantes, bajo los parámetros de modelos educativos constructivistas y colaborativos (Tejedor, F. y GarciaValcárcel, A. y Prada, S. 2009).

Una característica importante de este modelo pedagógico constructivista social es que está centrado en los estudiantes, quienes coordinan entre sí para realizar un diseño o proyecto con el que construirán nuevos conocimientos, reflexionarán sobre su entendimiento y desarrollarán destrezas y habilidades de alto orden. El profesor se convierte en un facilitador, debe diseñar estrategias y experiencias de aprendizaje según las características y necesidades de los estudiantes; por lo tanto, el docente 
debe ser cuidadoso al seleccionar la información, lo que el estudiante puede retener y significar a partir de su experiencia y los contenidos que ya posee, para establecer puentes cognoscitivos con la nueva información;

En relación con los recursos que ofrece el AV a sus usuarios, y la forma en que estos se definen, lo podemos observar en el siguiente cuadro.

Tabla 1. Recursos ofrecidos a los usuarios en el Aula Virtual

\begin{tabular}{|c|c|}
\hline Recursos & Descripción \\
\hline $\begin{array}{l}\text { Recursos trans- } \\
\text { misivos }\end{array}$ & $\begin{array}{l}\text { Se refiere a todos los } \\
\text { módulos, recursos y } \\
\text { actividades que tie- } \\
\text { nen como función } \\
\text { principal transmitir } \\
\text { información. }\end{array}$ \\
\hline $\begin{array}{l}\text { Recursos inte- } \\
\text { ractivos }\end{array}$ & $\begin{array}{l}\text { Hacen referencia al } \\
\text { control de navegación } \\
\text { por parte de los es- } \\
\text { tudiantes, sobre los } \\
\text { contenidos. }\end{array}$ \\
\hline $\begin{array}{l}\text { Recursos cola- } \\
\text { borativos }\end{array}$ & $\begin{array}{l}\text { Estas herramientas } \\
\text { posibilitan la disposi- } \\
\text { ción de recursos orien- } \\
\text { tados a la interacción, } \\
\text { el intercambio de ideas } \\
\text { y materiales tanto en- } \\
\text { tre el profesor y los es- } \\
\text { tudiantes como de los } \\
\text { estudiantes entre sí. }\end{array}$ \\
\hline $\begin{array}{l}\text { Herramientas de } \\
\text { comunicación }\end{array}$ & $\begin{array}{l}\text { Uno de los propósitos } \\
\text { principales de la plata- } \\
\text { forma consiste en faci- } \\
\text { litar y enriquecer la in- } \\
\text { teracción entre todos } \\
\text { sus usuarios a través } \\
\text { de Correo Electrónico, } \\
\text { Chats, Mensajes, Con- } \\
\text { sultas y Encuestas. }\end{array}$ \\
\hline
\end{tabular}

\section{Ventajas del uso del aula virtual}

En la práctica, el AV de un curso permite a los estudiantes el acceso a los cursos con total libertad más allá del horario dedicado a la asignatura, proporciona un entorno de aprendizaje y trabajo cooperativos, y posibilita la gestión de actividades académicas en comunidades virtuales, distribuye la información de forma rápida y precisa a todos los participantes; también posibilita enviar preguntas concretas o participar en grupos de discusión, navegar a través de las páginas electrónicas del curso y disponer de bibliografia, material didáctico, simulaciones y videos, trabajar un foro, intercambiar archivos, y comunicarse ya sea de forma sincrónica o asíncrona. Todo ello a fin de reforzar conceptos y mantener vivo el interés y la motivación por la investigación en los contenidos de la asignatura y, lo más importante, preparar al educando para competir en el mercado de manera ágil, rápida y eficiente. El material que en estos ambientes se presente debe ser cuidadosamente elaborado. (Reyes, K. 2007)

El AV puede constituirse en un buen aliado de una pedagogía activa, en buenos socios de aprendizaje significativo. Todo depende de que docentes y estudiantes perciban esta tecnología como nuevos medios de apoyo para aprender, como un poderoso potencial para la construcción de conocimientos y de cómo, para qué, cuándo, con qué y por qué usamos esta tecnología en el aprender.

Es necesario mencionar que el AV es una herramienta que amplifica la 
práctica pedagógica; sin embargo, su sola presencia no implica necesariamente una evolución de esta práctica, ya que se exige también la renovación de las competencias de los usuarios.

\section{Acciones fundamentales e impres- cindibles para el adecuado uso de las aulas virtuales}

Los vacíos en la formación de los docentes en medios y nuevas tecnologías de la información y la comunicación constituyen otra de las grandes barreras que limita o inhiben el uso de los mismos en las prácticas educativas, o al mal uso de los mismos en los procesos de enseñanza y aprendizaje. Esto les impide saber poseer un vasto marco referencial de cómo insertar los medios en el acto educativo, lo cual implica que los profesores presenten dificultades en las prácticas pedagógicas con los medios tecnológicos, tales como: no saber usarlos y no conocer sus aplicaciones educativas. (Romero, R. 2002)

Podemos concretar cuatro lineas de acciones fundamentales e imprescindibles para el adecuado uso de las AV:

\section{a) A Nivel de Autoridades}

Es imprescindible una toma de postura concreta y práctica por parte de las autoridades educativas de la universidad. Es fundamental la dotación de los equipos tecnológicos necesarios. Facilitar las conexiones con la red, los planes de capacitación de los docentes, favorecer todas las iniciativas para que el aprendizaje sea generalizado, aumentar los presupuestos para materiales de equipo, mantenimiento y formación. Se deben crear puestos de docentes en informática educativa, inspectores responsables de las Tecnologías de la Información y un responsable de la Tecnología Educativa.

\section{b) E1 Equipo Directivo}

No puede haber innovación educativa sin un proyecto, sin una planificación, sin la orientación y el apoyo de las autoridades académicas. Tratar de utilizar el AV en una institución educativa que no cuente con el apoyo y la planificación de la Alta Dirección es caminar hacia el fracaso. El apoyo se inicia con el convencimiento, de que nos encontramos ante una nueva situación y un nuevo paradigma y que hay que afrontar un cambio de enfoque en el que debemos participar todos. Por lo tanto, los Equipos Directivos deben apoyar a los docentes más motivados, animar a los dudosos y resolver las dificultades de los profesores renuentes y negativos.

\section{c) Infraestructura técnica/conexión a la red.}

No se puede poner en práctica el aprendizaje con un AV si la institución no cuenta con la estructura informática y de red necesarias. Evidentemente hay distintos niveles de equipamiento que pueden alcanzarse progresivamente. No se puede esperar a tener "todos" los equipos posibles y las condiciones óptimas para comenzar. Pero sí hace falta contar con los elementos tecnológicos imprescindibles para utilizar Internet en el aula. 


\section{d) La formación de los docentes}

La mejor manera de lograr el uso de esta tecnología entre los docentes, es promoviendo la adecuada formación desde el propio campus, incentivando el uso y la integración del AV a partir de la consideración de sus necesidades, orientada a la acción práctica (para que no se quede solo en teoría) y, facilitando los adecuados medios tecnológicos y un buen asesoramiento continuo.

Este es un objetivo no muy fácil de lograr, debido a que aún hay muchos docentes que ven con recelo e indiferencia el uso de estos recursos. Como bien expresa Marques, P. (2000), cambiar un paradigma exige una readaptación mental y estructural a las nuevas condiciones y al nuevo contexto. En esta dinámica de cambio y adaptación, como hemos mencionado anteriormente, no hay un solo punto de partida, son varios, y los impulsores de la innovación educativa deben actuar simultáneamente. Si falla uno de los elementos la acción queda gravemente lastrada. El problema aumenta cuando pensamos y comprobamos la dificil coordinación de cada una de las acciones que consideramos deben aunarse.

\section{Percepción de los estudiantes sobre el uso del aula virtual}

Las valoraciones personales de los estudiantes respecto a las interacciones con el AV como apoyo a las clases presenciales que favorezca su aprendizaje son importantes. Esta valoración funciona como motor de gratificación, pues obliga al estudiante a adaptarse al medio a través de la construcción de conceptos y uso del lenguaje en el ejercicio comunicativo. Por otro lado, también funciona como un contexto al que el estudiante apela para recordar, recrear y resignificar el contenido cuando está fuera de esa clase, ya que evocar lo sucedido en el seno del ámbito social de clase, los diálogos, las argumentaciones, etc. permiten al estudiante recontextualizar el contenido en futuras ocasiones.

Para indagar la valoración que hacen los estudiantes sobre el uso del $\mathrm{AV}$, debemos tener en cuenta que nuestros estudiantes actuales son hablantes nativos del lenguaje de las computadoras, los videojuegos e internet. Por el contrario, para muchos de los docentes, lo digital es una segunda lengua que se refleja fundamentalmente en nuestra vida académica $\mathrm{y}$ profesional. Usamos internet cuando necesitamos un libro que previamente dé cuenta del problema, antes de usar un aparato leemos el manual, antes de ejecutar un programa necesitamos saber que tecla tocar, etc. Justo a la inversa de los nativos digitales, que hacen primero y se preguntan después. Neurológicamente, este segundo lenguaje ocupa áreas del cerebro distintas de las que se movilizan con el aprendizaje de la lengua materna.

Debido a que pensamos que los estudiantes son nativos digitales, en muchos casos damos por sentado que el estudiante posee unas minimas competencias tecnológicas, pero no resulta tan obvio presuponer que cuentan con las habilidades necesarias para el aprendizaje autónomo. La adquisición de esas habilidades es el primer esca- 
lón que debe alcanzar, y para ello debe ir acompañado de un docente y una institución académica que facilite los recursos y establezca las condiciones y los contextos propicios para esa capacitación previa.

Sabiendo que el A V está conformado por un conjunto de herramientas y como tales requieren de ciertas habilidades para ser utilizadas, estas habilidades están condicionadas por el conocimiento previo del medio, el grado de acceso a la tecnología, en calidad y frecuencia, y los usos posibles que se perciben en relación con estos medios. De esta manera, a la hora de adoptar el AV para la educación, es recomendable que el estudiante esté investido de las siguientes destrezas: Conocer cuándo hay una necesidad de información, identificar esta necesidad, saber trabajar con diferentes fuentes y sistemas simbólicos, dominar la sobrecarga de información, evaluarla y discriminar su calidad, organizarla, tener habilidad para la exposición de pensamientos, ser eficaz en el uso de la información para dirigir el problema, y saber comunicar a otros la información encontrada.

Por otro lado, cuando se introduce un nuevo medio dentro de un repertorio de prácticas es necesario corroborar si contribuye a la buena enseñanza. E1 uso de los medios más avanzados en la enseñanza no garantiza la motivación del estudiante. Un aspecto crucial en la evaluación del medio es la percepción de los estudiantes que se enfrentan a esta innovación, la cual puede potenciar o anular los objetivos intelectuales y actitudinales previstos en la asignatura, así como ocasionar cambios no previstos.

Para poder aprovechar al máximo el potencial que ofrece el AV para los estudiantes, han de cumplirse requisitos fundamentales, tales como:

a) Concientización a los estudiantes sobre su responsabilidad y participación en su aprendizaje. La explicitación de los objetivos de aprendizaje a los estudiantes redunda en múltiples ventajas pedagógicas importantes: ayuda a centrar la atención en las tareas venideras, lo que fomenta su motivación; así como asumir una verdadera corresponsabilidad en su propio proceso de aprendizaje, con todo lo que esto supone: elecciones con conocimiento de causa, valoración de su propio aprendizaje, evaluación de sí mismo en tanto que aprendiz, o diálogo realmente interactivo con el docente sobre este tema. Esta observación de carácter general también es aplicable al aprendizaje a través del AV.

b) Participación frecuente. Tanto el docente como el estudiante deben estar dispuestos a establecer una colaboración vinculante respecto al aprendizaje en una asignatura. Mediante una participación frecuente (leyendo y escribiendo) en los foros electrónicos, ambas partes asumen una corresponsabilidad en el éxito del uso del AV. Algunas actividades en el AV pueden ir acompañadas de fechas limite de cumplimiento obligado, tanto para el docente como para el estudiante. Es preciso establecer en todo momento directrices claras so- 
bre el uso del AV y hallar maneras de asegurar su cumplimiento.

c) Cumplimiento de plazos establecidos. El cumplimiento de los plazos establecidos es tan importante en el AV como en el aula presencial, tanto si se trabaja individualmente como en grupo. Es una ventaja acordar de antemano la fecha límite para publicar un mensaje en una conferencia, así como animar a que los plazos convenidos sean cumplidos por todas las partes. En la planificación de un programa coherente de aprendizaje electrónico, el incumplimiento de los plazos establecidos puede ser un obstáculo para su desarrollo y, por tanto, para el éxito previsto. En caso de que el docente o el estudiante, no pudiera cumplir un plazo establecido, por respeto a los demás participantes debería notificarlo en un mensaje, indicando a la vez para cuándo puede publicar el mensaje.

d) Finalmente, evaluación de la percepción de los usuarios del medio, para determinar cuáles son las fallas que ellos notan, ya que esta tecnologia se ha incorporado para apoyar el aprendizaje de los estudiantes. (Cabero, J. 2009)

\section{MÉTODO}

Se planteó una investigación de tipo descriptivo, no experimental de corte transversal, ya que se han tomado los datos en un solo momento en el tiempo, a partir de un muestreo no probabilístico. Para medir la per- cepción de los estudiantes de la universidad sobre la utilidad de las aulas virtuales para el aprendizaje, se administró un cuestionario a 722 estudiantes en las facultades de Ciencias Económicas y Empresariales, e Ingeniería. Este instrumento, constó de diversas preguntas, con las siguientes opciones de respuesta: Muy de acuerdo (MDA), De acuerdo (DA), Ni de acuerdo, ni en desacuerdo (NA, ND), En desacuerdo (ED), Muy en desacuerdo (MD) y No sabe, no responde $(\mathrm{Ns} / \mathrm{Nr})$, que intentan establecer la percepción de los usuarios de esta herramienta tecnológica. Dichas preguntas se han clasificado en dos categorias: la percepción por parte de los estudiantes sobre la utilidad del AV para la enseñanza y la percepción sobre las actividades que realizan los docentes a través del AV, lo cual facilita el análisis y comprensión de los datos presentados.

\section{RESULTADOS OBTENIDOS}

El análisis se enfoca básicamente en dos aspectos: la percepción por parte de los estudiantes sobre la utilidad de aulas virtuales para la enseñanza, y la apreciación que tienen sobre las actividades que realizan los docentes en las aulas virtuales.

A continuación, se muestran los resultados:

\section{La percepción por parte de los es- tudiantes sobre la utilidad de aulas virtuales para la enseñanza.}

Tal como se muestra en la tabla 2, el $44.88 \%$ de los estudiantes conside- 
ran que el AV incrementa muy poco el interés por los temas propuestos en las clases; el 46.4\% de los estudiantes opinan que las actividades que los docentes proponen en el AV son muy poco motivadoras; el $34.07 \%$ piensan que el AV facilita bastante la comunicación con sus compañeros de clase para realizar tareas en equipo; el $36.43 \%$ opinan que el aula virtual es muy poco útil para una mayor comunicación con sus profesores al realizar consultas y obtener asesoramiento; sin embargo, un $26.73 \%$ consideran muy útil el AV para este fin; el 43.21\% consideran que el AV es casi nada útil como complemento para entender mejor las clases; y el 50\% consideran que en el AV son muy pocos los recursos que el docente utiliza.

Tabla $\mathbf{N}^{\circ}$ 2. Percepción de los estudiantes respecto al aula virtual

\begin{tabular}{lcccc}
\hline & Bastante & Poco & Muy poco & Casi nada \\
\hline $\begin{array}{l}\text { Crees que el aula virtual incrementa } \\
\text { el interés por los temas propuestos en } \\
\text { las clases }\end{array}$ & $4.43 \%$ & $8.17 \%$ & $44.88 \%$ & $42.52 \%$ \\
$\begin{array}{l}\text { Te sientes más motivado cuando tus } \\
\text { profesores proponen actividades a tra- }\end{array}$ & $6.51 \%$ & $8.17 \%$ & $46.40 \%$ & $38.92 \%$ \\
$\begin{array}{l}\text { vés del aula virtual } \\
\begin{array}{l}\text { El aula virtual te facilita la comunica- } \\
\text { ción con tus compañeros de clase para }\end{array} \\
\text { realizar tareas en equipo. }\end{array}$ & & & \\
$\begin{array}{l}\text { El aula virtual te permite una mayor } \\
\text { comunicación con tus profesores para } \\
\text { realizar consultas y obtener asesora- } \\
\text { miento. }\end{array}$ & $26.73 \%$ & $19.67 \%$ & $36.43 \%$ & $17.17 \%$ \\
$\begin{array}{l}\text { Encuentras en el aula virtual un com- } \\
\text { plemento para entender mejor las cla- } \\
\text { ses. }\end{array}$ & $5.96 \%$ & $11.63 \%$ & $39.52 \%$ & $15.65 \%$ \\
$\begin{array}{l}\text { Los recursos que el profesor utiliza en } \\
\text { el aula virtual son variados. }\end{array}$ & $4.57 \%$ & $15.10 \%$ & $50.00 \%$ & $30.33 \%$ \\
\hline
\end{tabular}

En cuanto a la pregunta si las actividades que realiza en el aula virtual le han ayudado a obtener mejores calificaciones, el 62.05\% manifiestan que sí le han ayudado a obtener mejores calificaciones, mientras que el 37.95\% mencionaron que estas actividades no le ayudaron a mejorar las calificaciones. 
Tabla $\mathrm{N}^{\circ}$ 2.1. Las actividades que realizas en el aula virtual te han ayudado a obtener mejores calificaciones

\begin{tabular}{lcr}
\hline & Frecuencia & Porcentaje \\
\hline No & 274 & $37.95 \%$ \\
$\mathrm{Si}$ & 448 & $62.05 \%$ \\
\hline Total & 722 & $100.00 \%$ \\
\hline
\end{tabular}

Percepción sobre el uso que hacen los docentes del Aula Virtual

En la tabla 3, se observa que el $21.05 \%$ de los estudiantes encuestados opina que el docente usa bastante el AV; el 26.04\% considera que el docente usa poco el aula virtual; el $20.36 \%$ opina que el aula virtual es muy poco utilizado por el docente; y el $32.55 \%$ consideran que el aula virtual casi no es usado por el docente.

Tabla $\mathbf{N}^{\circ}$ 3. Percepción de los estudiantes respecto al aula virtual en porcentajes

\begin{tabular}{lll}
\hline & Frecuencia & Porcentaje \\
\hline Bastante & 152 & $21.05 \%$ \\
Poco & 188 & $26.04 \%$ \\
Muy poco & 147 & $20.36 \%$ \\
Casi nada & 235 & $32.55 \%$ \\
Total & 722 & $100 \%$ \\
\hline
\end{tabular}

En lo referente a las actividades que realizan los docentes en el AV, se aprecia en la tabla 3.1. que los docentes utilizan frecuentemente el AV para enviar documentos office, como también para enviar tareas y ejercicios a sus estudiantes; así también, se ob- serva que no es muy frecuente el uso del AV para asesorar y/o guiar a los estudiantes en lo que respecta a las actividades, proponer investigaciones, realizar autoevaluaciones y programar foros para discusión de temas o problemas relacionados con los temas desarrollados en clase son imperceptibles.

Tabla 3.1. Actividades realizadas por los docentes en el Aula Virtual Actividades del docente en el A.V.

Total Porcentaje

\begin{tabular}{lcc}
\hline $\begin{array}{l}\text { Envio de docu- } \\
\text { mentos Office }\end{array}$ & 693 & $96.26 \%$ \\
$\begin{array}{l}\text { Enlaces a páginas } \\
\text { web }\end{array}$ & 438 & $60.66 \%$ \\
Asesoramiento & 294 & $40.72 \%$ \\
Tareas & 602 & $83.38 \%$ \\
Ejercicios & 570 & $78.95 \%$ \\
Investigación & 21 & $2.91 \%$ \\
Autoevaluación & 18 & $2.49 \%$ \\
Foro & 11 & $1.52 \%$ \\
\hline
\end{tabular}

Relación entre percepción que tienen los estudiantes sobre el AV y el tipo de facultad a la que pertenecen.

En la tabla 4, se observa la distribución entre las categorias de las variables investigadas. Así también, los totales de las categorías para cada variable. Se puede ver que la percepción del estudiante respecto al AV es similar en ambas facultades, y no se observa diferencias. 
Tabla $\mathbf{N}^{\circ}$ 4. Tipo de facultad vs percepción de los estudiantes

\begin{tabular}{|l|l|l|l|l|l|l|}
\hline \multirow{2}{*}{\multicolumn{2}{|c|}{}} & \multicolumn{4}{|c|}{ Percepción de los estudiantes } & \multirow{2}{*}{ Total } \\
\cline { 3 - 7 } & Deficiente & Regular & Buena & $\begin{array}{c}\text { Muy } \\
\text { buena }\end{array}$ & \\
\hline \multirow{2}{*}{ Facultad } & INGENIERIA & $55(16 \%)$ & $186(53 \%)$ & $97(28 \%)$ & $10(3 \%)$ & $348(100 \%)$ \\
\cline { 2 - 7 } & CC.EE. y E & $52(14 \%)$ & $212(57 \%)$ & $98(26 \%)$ & $12(3 \%)$ & $374(100 \%)$ \\
\hline Total & 107 & 398 & 195 & 22 & $\mathrm{n}=722$ \\
\hline
\end{tabular}

Relación entre el uso por los docentes del AV. y el tipo de facultad a la que pertenecen los estudiantes

En la tabla 4.1, se observa la distribución entre las categorias de las variables investigadas. Así también, los totales de las categorias para cada variable. Se puede ver que el uso que hace el docente del AV, es casi similar en ambas facultades.

Tabla $\mathrm{N}^{\circ}$ 4.1. Tipo de facultad vs uso del AV por los docentes

\begin{tabular}{|c|c|c|c|c|c|c|}
\hline \multicolumn{2}{|c|}{} & \multicolumn{4}{c|}{ Uso del AV por los docentes } & \multirow{2}{*}{ Total } \\
\cline { 3 - 6 } \multicolumn{2}{|c|}{} & Muy bajo & Bajo & Parcial & Alto & \\
\hline \multirow{2}{*}{ Facultad } & INGENIERIA & $21(6 \%)$ & $51(15 \%)$ & $\mathbf{1 8 7}(\mathbf{5 4 \% )}$ & $89(26 \%)$ & $348(100 \%)$ \\
\cline { 2 - 6 } & CC.EE. & $31(8 \%)$ & $58(16 \%)$ & $102(27 \%)$ & $\mathbf{1 8 3}(\mathbf{4 9} \%)$ & $374(100 \%)$ \\
\hline \multicolumn{2}{|c|}{ Total } & 52 & 109 & 289 & 272 & 722 \\
\hline
\end{tabular}

En la tabla 4.2, es posible observar la distribución entre las categorías de las variables investigadas. Así mismo, los totales de las categorías para cada variable. Se puede ver que el uso total y parcial del AV por parte del docente, tiene una mayor frecuencia. Por otro lado, la apreciación del estudiante respecto al uso que hace el docente del AV, es mayormente que los docentes hacen un uso regular y, en un segundo lugar están los que perciben que hay una buena participación de los docentes en el AV. 
Tabla $\mathbf{N}^{\circ}$ 4.2. Tabla cruzada entre la percepción del estudiante y el uso del Aula Virtual por parte de los docentes

\begin{tabular}{|c|c|c|c|c|c|}
\hline \multirow{2}{*}{$\begin{array}{c}\text { Uso del AV por el } \\
\text { docente }\end{array}$} & \multicolumn{4}{|c|}{ Percepción del estudiante } & \multirow{2}{*}{ Total } \\
\cline { 2 - 5 } & Deficiente & Regular & Buena & Muy buena & \\
\hline Muy bajo & $10(19.23 \%)$ & $26(50 \%)$ & $11(21.15 \%)$ & $5(9.62 \%)$ & 52 \\
\hline Bajo & $5(4.59 \%)$ & $68(62.39 \%)$ & $30(27.52 \%)$ & $6(5.5 \%)$ & 109 \\
\hline Parcial & $44(15.22 \%)$ & $154(53.29 \%)$ & $86(29.76 \%)$ & $5(1.73 \%)$ & 289 \\
\hline Alto & $48(17.65 \%)$ & $150(55.15 \%)$ & $68(25 \%)$ & $6(2.21 \%)$ & 272 \\
\hline TOTAL & $107(15 \%)$ & $398(55 \%)$ & $195(27 \%)$ & $22(3 \%)$ & $\mathrm{n}=722$ \\
\hline
\end{tabular}

\section{CONCLUSIONES}

En relación a los objetivos del estudio y las hipótesis planteadas, se llegó a las siguientes conclusiones:

Los estudiantes consideran que el AV incrementa muy poco el interés por los temas propuestos en las clases; las actividades que los docentes proponen son muy poco motivadoras; no la consideran útil como complemento para entender mejor las clases, y aunque son muy pocos los recursos que el docente utiliza, sí les ha ayudado a mejorar sus calificaciones.

Los estudiantes opinan que los docentes utilizan el AV para enviar documentos office, solicitar tareas y ejercicios, pero no la usan para asesorar y/o guiar a los estudiantes; tampoco proponen investigaciones, autoevaluaciones y foros de discusión de problemas relacionados con los temas desarrollados en clase.

No se ha encontrado diferencia significativa entre la percepción que tienen los estudiantes de la Facultad de Ciencias Económicas y Empresariales y de la Facultad de ingeniería sobre el AV.

En cuanto a la opinión sobre el uso que hacen los docentes del AV, sí hay diferencia: los estudiantes de la facultad de Ciencias Económicas y Empresariales opinan que los docentes hacen un uso alto del AV, mientras que los estudiantes de la facultad de Ingeniería señalan que los docentes hacen un uso parcial del AV. 


\section{REFERENCIAS BIBLIOGRÁFICAS}

Cabero, J. \& Ot. (2000). Los usos de los medios audiovisuales, informáticos y las nuevas tecnologias en los centros andaluces. Texto original en: Y continuamos avanzando. Las nuevas tecnologías para la mejora educativa. Sevilla: Editorial Kronos, S.A, pp. 467-558.

Escalona, P.; Rodríguez, E. \& García, R. (2013). El Moodle, una plataforma de apoyo al aprendizaje colaborativo. Recuperado de http://revistas.mes.edu.cu/ greenstone/collect/repo/index/assoc/. Consulta: 12-05-14

Morales, L. \& Guevara J. (2009). Actitudes de los Estudiantes y Docentes hacia la Computadora y los Medios para el Aprendizaje. México: ILCE.

Marqués, P. (2000). Criterios para la clasificación y evaluación de espacios web de interés educativo. Localización: Educar, ISSN 0211-819X, N² 25, Págs. 95-111.

Reyes, K. (2007). Aula Virtual basada en la teoria constructivista empleada como apoyo para la enseñanza de los sistemas operativos a nivel universitario. Escuela de Ingeniería de Sistemas y Computación. Universidad Sto. Toribio de Mogrovejo. Chiclayo.

Romero, R. (2002): Posibilidades y limitaciones de la integración de los medios de enseñanza. Recuperado en: http://tecnologiaedu.us.es/revistaslibros / public28.html] Consulta: 22-06-14.

Sánchez, J. \& Ramos, F. (2012). Usos pedagógicos de Moodle en la docencia universitaria desde la perspectiva de los estudiantes. Revista Iberoamericana de Educación 60 (1): 15-38.

Sulmont, L. (2007). El valor pedagógico del Aula Virtual: Hablan los usuarios. Revista Docencia Universitaria. Año 3-N²-, Universidad Peruana de Ciencias Aplicadas. Lima.

Tejedor, F.; García-Valcárcel, A. \& Prada, S. (2009). Medida de actitudes del profesorado universitario hacia la integración de TIC. Comunicar, $\mathrm{N}^{\circ} 33$, V. XVII, 2009, Revista Científica de Educomunicación; ISSN: 1134-3478; páginas 115124. España. 